not required thyroid replacement therapy during or for 9 years before this pregnancy. She was taking daily antidepressants for major depression. Birth complications included meconium in the amniotic fluid, cesarean section for abnormal fetal heart rate, neonatal respiratory distress, and treatment in the neonatal intensive care unit. The Apgar scores were 8 and 9 at 1 and 5 minutes. Birth weight was at the $10-25^{\text {th }} \%$, length $<10^{\text {th }} \%$, and head circumference of $34 \mathrm{~cm}\left(25-50^{\text {th }} \%\right)$. Cranial sutures were wide and the posterior fontanel measured $4 \mathrm{~cm}$. Oxygen saturation was $95 \%$, and respiratory distress resolved in a few hours. Rhythmic jerking started in the right arm on day 6 and became generalized. Seizures were unresponsive to an initial phenobarbital bolus, and were controlled by addition of lorazepam and phenytoin. Lumbar puncture showed normal CSF, and blood electrolytes, glucose, and amino acids and urine organic acids were normal. EEG, CT scan, and MRI were normal. Thyroid function studies on day 9 were as follows: TSH $0.67(\mathrm{~N}: 0.4-4.7 \mathrm{uIU} / \mathrm{mL})$; free T4 0.5 (N: 0.7-1.8 ng/dL); free T3 $1.41(\mathrm{~N}: 1.5-3.5 \mathrm{pg} / \mathrm{mL})$. On day 15 after L-thyroxine 25 ug/day, the TSH was 0.71 and free T4 1.4. Maternal TSH and T4 were normal. Seizures stopped and antiepileptic medications were discontinued, with one relapse at 2 months associated with hypothyroidism (hoarse cry, wide fontanel, delayed bone age, temperature instability). Seizures responded to an increase in thyroxine. The neurologic evaluation at 6 months follow-up was normal. (Aly H, Kanter DE, Fisher-Owens SA. J Pediatr Neurol 2004;2:111-113). (Respond: Hany Aly MD, $90023^{\text {rd }}$ Street, NW Room G-132, Washington, DC 20037).

COMMENT. Multiple factors may have contributed to the seizures in this case (hypoxic-ischemic encephalopathy, maternal diabetes and antidepressants) in addition to hypothyroidism. The authors argue against alternative causes and find the response to thyroxine and successful withdrawal of AEDs to be strongly supportive of an association. The expected rise in TSH with congenital hypothyroidism may be delayed in some newborns, and the initial low TSH and low free T4 are compatible with the diagnosis. (Nelson Pediatrics gives newborn normal values: TSH 1.0-9.1 and T4 2.0-4.9). The routine neonatal metabolic screening may be normal, as in this infant, and this finding should not discourage further testing for possible hypothyroidism as a cause of neonatal seizures refractory to therapy. Seizures are known to complicate myxedema in adults but are rare with congenital hypothyroidism.

\title{
PHOTOSENSITIVE EPILEPSY
}

The evolution and disappearance of photosensitivity (PS) was studied long-term in 42 patients (17 males, 25 females; mean age at onset 6 years 9 months, range 5 to 12 years) with electroencephalographic evidence of photosensitive epilepsy at University of Chieti, San Valentino Hospital, and Brindisi Hospital, Italy. Valproate (VPA) monotherapy was begun after the second seizure in 36 patients, VPA with carbamazepine or lamotrigine in 4 , and stimuli avoidance but no drugs in 2. Mean duration of follow-up was 8 years 1 month, and the mean age at end of follow-up was 15 years 2 months. At end of follow-up, photoparoxysmal responses (PPRs) were present in 25 and had disappeared in 15 . Of 31 (75\%) responding to antiepileptic drugs (AEDs), 19 had persistent PS and 12 were PS free. Photosensitive epilepsy has a good prognosis for seizure control, independent of persistence or disappearance of PS. Response to AEDs is not dependent on disappearance of PS. PS is 
probably not linked to a genetic susceptibility to epilepsy. (Verrotti A, Trotta D, Salladini C et al. Photosensitivity and epilepsy: a follow-up study. Dev Med Child Neurol 2004;46:347351). (Respond: Alberto Verrotti MD, PhD, Department of Medicine, Section of Pediatrics, University of Chieti-Ospedale Policlinico, Via dei Vestini 5, 66100 Chieti, Italy).

COMMENT. Photosensitive epilepsy is defined as recurrent seizures in photosensitive patients, with EEG-PPRs in response to intermittent photic stimulation. According to several references cited by the authors, photosensitivity (PS) occurs in 2 to $20 \%$ of patients with epilepsy, and may also be present in non-epileptic individuals. PS can occur in any type of epileptic disorder and is not a specific epileptic syndrome. Pure PS epilepsy is characterized by generalized paroxysmal epileptiform discharges exclusively provoked by flicker, usually in adolescence, predominantly females. PS epilepsy has a good prognosis, independent of disappearance or persistence of PS

Short-term outcome of Pokemon phenomenon and TV-induced seizures was determined at Nagoya University, Japan (Ishiguro Y, Takada H, Watanabe K et al. A follow-up survey on seizures induced by animated cartoon TV program "Pocket Monster." Epilepsia April 2004;45:377-383). Of 103 patients with epileptic seizures during the TV program, 25 (24\%) had unprovoked seizures before the incident (Epilepsy group), and 78 (76\%) did not (Nonepilepsy group). Twenty-three (22\%) had seizures after the incident, 15 visually induced. Patients in the Epilepsy group had more seizure recurrences (both unprovoked or visually induced) than the Non-epilepsy group ( $56 \%$ vs $9 \%$; p $<0.0001)$. PPRs on EEG were present in $45(46 \%)$ patients, and spontaneous epileptiform discharges in $49(50 \%)$. PPRs were not correlated with recurrence of seizures, whereas spontaneous epileptiform discharges were significantly correlated $(34 \%$ vs $8 \% ; \mathrm{p}<0.01)$. Of patients with seizures during the TV incident, $70(68 \%)$ had no seizures before or during the 3 year follow-up. Color changes, specifically red, rather than luminance was found to be the critical seizure-inducing factor in the TV scene, prompting color restrictions in TV cartoon programs in Japan.

For reports and commentaries on visually-induced seizures, including video game seizures and pattern sensitive seizures, see Progress in Pediatric Neurology III, PNB Publishers, 1997;64-67.

\section{SLEEP DISORDERS}

\section{DIAGNOSIS AND MANAGEMENT OF CHILDHOOD NARCOLEPSY}

The clinical and polysomnographic presentation of 12 published cases of narcolepsy with onset from early infancy to 8 years is reviewed at Victoria University, Melbourne, and Flinders Medical Centre, Adelaide, Australia. Diagnosis was made at ages 2 years to 12 years. Clinical presentations included excessive daytime sleepiness (EDS) in all cases, cataplexy in 6, behavioral problems and/or inattention in 6, hypnogogic hallucinations in 3 , and sleep paralysis in 1. Alternate diagnoses considered in 5 patients were epilepsy (3), comorbid ADHD and ODD (2), developmental delay (2), obstructive sleep apnea (1), and Munchausen syndrome by proxy (1). Sleepiness in narcolepsy persists despite adequate nocturnal sleep and it occurs in inappropriate environments, while eating, watching TV, playing, and even walking. Persistence of daytime napping after 4 years of age may be an 